The Astrophysical Journal, 525:L53-L56, 1999 November 1

(C) 1999. The American Astronomical Society. All rights reserved. Printed in U.S.A.

\title{
THE CIRCUMSTELLAR DISK OF HD 141569 IMAGED WITH NICMOS
}

\author{
A. J. Weinberger, ${ }^{1}$ E. E. Becklin, ${ }^{1}$ G. Schneider, ${ }^{2}$ B. A. Smith, ${ }^{3}$ P. J. Lowrance, ${ }^{1}$ \\ M. D. Silverstone, ${ }^{1}$ B. Zuckerman, ${ }^{1}$ and R. J. Terrile ${ }^{4}$ \\ Received 1999 August 6; accepted 1999 September 3; published 1999 October 5
}

\begin{abstract}
Coronagraphic imaging with the Near-Infrared Camera and Multiobject Spectrometer on the Hubble Space Telescope reveals a large, $400 \mathrm{AU}\left(4^{\prime \prime}\right)$ radius, circumstellar disk around the Herbig Ae/Be star HD 141569. A reflected light image at $1.1 \mu \mathrm{m}$ shows the disk oriented at a position angle of $356^{\circ} \pm 5^{\circ}$ and inclined to our line of sight by $51^{\circ} \pm 3^{\circ}$; the intrinsic scattering function of the dust in the disk makes the side inclined toward us, the eastern side, brighter. The disk flux density peaks 185 AU (1".85) from the star and falls off to both larger and smaller radii. A region of depleted material, or a gap, in the disk is centered 250 AU from the star. The dynamical effect of one or more planets may be necessary to explain this morphology.
\end{abstract}

Subject headings: circumstellar matter — stars: emission-line, Be — stars: individual (HD 141569)

\section{INTRODUCTION}

The discovery of disks around main-sequence stars has opened up possibilities for studying nascent planetary systems. Old Herbig Ae/Be and $\beta$ Pictoris analog stars lie at a transitional age between pre-main-sequence and zero-age main-sequence stars. Both classes have infrared excesses indicative of the presence of warm circumstellar dust as well as luminosities and colors consistent with those of young stars. Their circumstellar material, in the form of disks, may be composed of both remnant protostellar and secondary "debris" dust generated by collisions.

HD 141569 (=IRAS 15473-0346 = SAO 140789), classified as B9.5 Ve by Jaschek \& Jaschek (1992), is a Herbig Ae/Be star with double-peaked $\mathrm{H} \alpha$ emission. Jaschek, Jaschek, \& Egret (1986) first identified it as a main-sequence star with infrared excess and a spectral energy distribution peaking at $\sim 60 \mu \mathrm{m}$. The disk has an optical depth of $\tau_{3-100 \mu \mathrm{m}}=$ $L_{\text {disk }} / L_{*}=8.4 \times 10^{-3} \quad$ (Zuckerman, Forveille, \& Kastner 1995), which is a few times larger than the two well-known infrared excess stars $\beta$ Pic and HR 4796A. With an Hipparcosmeasured distance of $99_{-8}^{+9} \mathrm{pc}$ (so $1^{\prime \prime} \approx 100 \mathrm{AU}$ ), the star may lie on the outskirts of the dark cloud complex L134/L134N (Sahu et al. 1998). Assuming its $B-V=0.095 \mathrm{mag}$ color is indicative of interstellar reddening, HD 141569 has an absolute magnitude of $M_{V}=1.6$ mag (van den Ancker, de Winter, \& Tjin A Djie 1998). Such a low luminosity for its spectral type is consistent with that of other young main-sequence A stars (Jura et al. 1998).

The age of HD 141569 is not well determined. It is located approximately on the zero-age main sequence, has an infrared optical depth similar to that of other young A stars, has strong CO emission (Zuckerman et al. 1995), and has two T Tauri-like companion stars (Weinberger et al. 1999). Together, these constrain its age to $10^{6}-10^{7} \mathrm{yr}$.

\footnotetext{
${ }^{1}$ Department of Physics and Astronomy, University of California, Los Angeles, Box 156205, Los Angeles, CA 90095-1562; alycia@astro.ucla.edu, becklin@astro.ucla.edu, lowrance@astro.ucla.edu, murray@astro.ucla.edu, ben@astro.ucla.edu.

${ }^{2}$ Steward Observatory, University of Arizona, 933 North Cherry Avenue, Tucson, AZ 85721-0065; gschneider@as.arizona.edu.

${ }^{3}$ Institute of Astronomy, 2680 Woodlawn Drive, University of Hawaii, Honolulu, HI 96822; brad@mahina.ifa.hawaii.edu.

${ }^{4}$ Jet Propulsion Laboratory, MS 183-503, Caltech, 4800 Oak Grove Drive, Pasadena, CA 91109; richard.j.terrile@jpl.nasa.gov.
}

The $1.1 \mu \mathrm{m}$ results reported here are part of our guaranteed time project to study the environments of nearby stars with the Near-Infrared Camera and Multiobject Spectrometer (NICMOS) aboard the Hubble Space Telescope (HST) (Schneider 1998). HD 141569 was included because of its large farinfrared optical depth, CO emission, and extended elliptical disk in mid-infrared images by Silverstone et al. (1999). The scattered-light disk around HD 141569 has also independently been observed with NICMOS at $1.6 \mu \mathrm{m}$ by Augereau et al. (1999a).

\section{OBSERVATIONS}

Coronagraphic observations of HD 141569 were made on 1998 September 27 with NICMOS Camera 2. The F110W filter $\left(\lambda_{\text {central }}=1.10 \mu \mathrm{m}, \mathrm{FWHM}=0.59 \mu \mathrm{m}\right)$ was used to maximize the angular resolution while minimizing scattered light. With the target positioned in the 0.3 radius coronagraph, three MULTIACCUM sequences were obtained for a total integration time of $607.9 \mathrm{~s}$ at each of two spacecraft orientations, rolled $8^{\circ}$ about the target axis with respect to each other. To minimize time-dependent point-spread function (PSF) variations, the two image sets were obtained within 40 minutes.

Prior to each set of coronagraphic observations, $0.36 \mathrm{~s}$ target acquisition frames were obtained with the $\mathrm{F} 171 \mathrm{M}\left(\lambda_{\text {central }}=\right.$ $1.72 \mu \mathrm{m}, \mathrm{FWHM}=0.07 \mu \mathrm{m})$ filter. Contemporaneous lamp flats and backgrounds were obtained at F160W for the purpose of locating the coronagraphic hole and enabling good flatfielding near the hole.

\section{DATA ANALYSIS}

The MULTIACCUM data sets were processed with the NICREDUCE software (McLeod 1997). Dark frames taken on 1998 September 20 were used to subtract the dark current and correct the detector shading. A special coronagraphic flat field was created by modifying a standard high signal-to-noise ratio on-orbit $\mathrm{F} 110 \mathrm{~W}$ reference flat field. Within a radius of 12 pixels $(0.91)$ of the hole, the pixel sensitivities were measured in the F160W hole-finding image, scaled to F110W, and replaced in the reference flat. After bias subtraction, linearization, and flatfielding, known bad pixels were corrected with a distanceweighted average within a radius of 8 pixels, and the three MULTIACCUM images from each spacecraft orientation were medianed. The best available photometric calibration was 


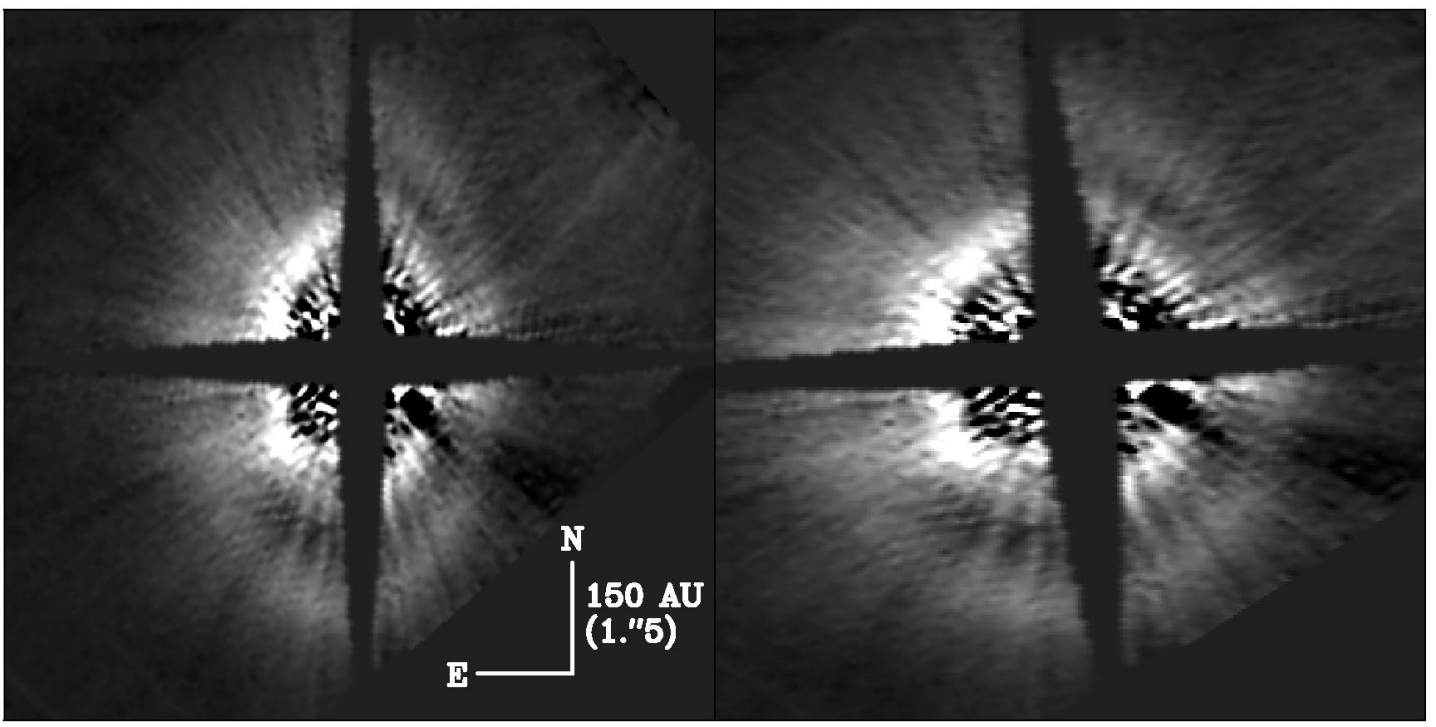

FIG. 1.-Left: Image of the reflected light disk around HD 141569 shown in a stretch that is linear with flux density. The eastern side of the disk is brighter because of the intrinsic scattering phase function of the dust. Right: Deprojected image of the disk using an inclination angle of $51^{\circ}$. Artifacts from small instabilities in the PSF subtraction process include the radial "streamers" and the dark "finger" in the inner disk at the 4 o'clock position. These residuals do not significantly affect the radial profile or the integrated flux density. The diffraction spikes narrow to higher radius as the overlap between the two sets of observations at different orientations increases and allows pixels behind the spikes in one image to be replaced by pixels from the other image.

applied to the final F110W images in which $1 \mathrm{ADU} \mathrm{s}^{-1}=$ $2.031 \times 10^{-6} \mathrm{Jy}$ and $0 \mathrm{mag}=1775 \mathrm{Jy}(\mathrm{M}$. Rieke 1999 , private communication).

In the reduced and calibrated NICMOS images, scattered light from the star still dominates the flux levels at radii of $1^{\prime \prime}-5^{\prime \prime}$, so in order to see the disk, a PSF star must be subtracted. The same observing strategy and the F110W filter were used for 14 other stars in this GTO program, and targets that do not show extended disk emission form a library of possible PSF

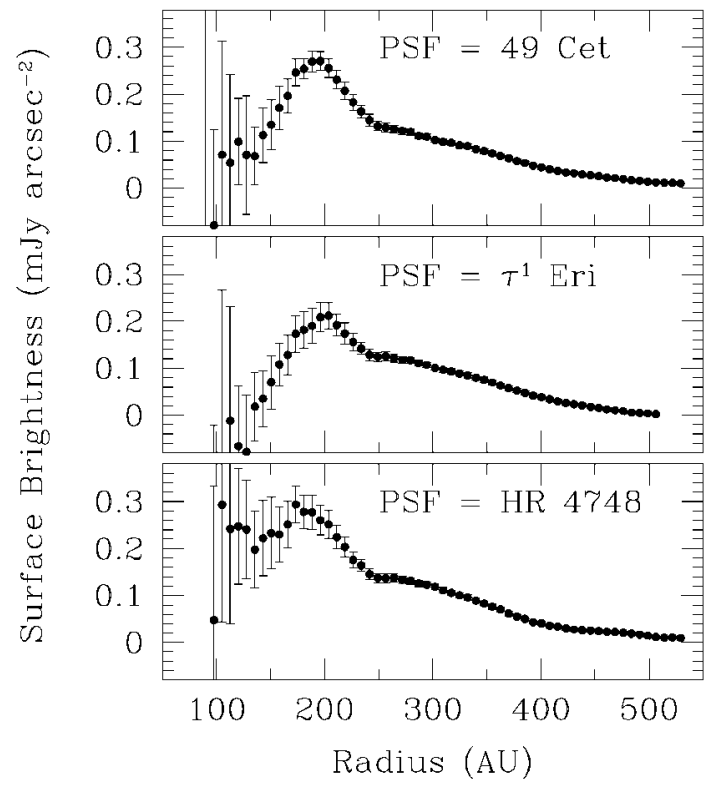

FIG. 2.-Azimuthally averaged surface brightness profile of the disk after the subtraction of three different PSF stars. The error bars represent only the statistical uncertainty in the mean surface brightness at each radius. At radii greater than $\sim 160 \mathrm{AU}$, the three profiles are consistent to within these uncertainties. The subtractions with $\tau^{1}$ Eri and 49 Cet suggest that the disk flux falls off substantially inside of $160 \mathrm{AU}$. stars. The detailed character of the NICMOS PSF changes with time primarily because of thermal variations in the HST optical assembly (Kulkarni et al. 1999); hence, some PSF star subtractions produce much lower residuals than others. Each PSF image was registered using cubic convolution interpolation to the images of HD 141569 at each spacecraft orientation. Only stars brighter than HD 141569 were considered, and the PSF subtraction residuals are dominated by systematic uncertainties.

Scattered light from two nearby stars outside the field of view (see § 5.1) pollutes the northwest corner of the image. For both roll angles, we used synthetic PSFs generated with Tiny Tim (Krist \& Hook 1997) and positioned them at the locations of the two stars in order to subtract this excess scatter. A small amount of residual flux, however, can still be seen in Figure 1.

Figure 2 compares the azimuthally averaged disk flux that was observed after the subtraction of each of the three bestmatched PSF stars. Two of these PSF stars, $\tau^{1}$ Eridani and 49 Ceti, were disk candidates in which no disk was detected, and the third, HR 4748, was observed specifically as a PSF star. At radii greater than 1"6 (160 AU), the morphology of the disk is consistent in all three subtractions. The subtraction of 49 Cet yielded the smallest amplitude residuals, and all further analysis was carried out on images using it as the PSF.

The PSF-subtracted images from each telescope roll were rotated to a common orientation and averaged. Regions of the image that were obscured by the diffraction spikes in one roll were replaced where possible by uncorrupted regions in the other roll.

\section{RESULTS}

The PSF-subtracted, roll-combined coronagraphic image of HD 141569 is shown in the left panel of Figure 1. An elongated disk is clearly evident at radii from 1".6 to 4" (160-400 AU) from the star. Elliptical isophotes were fitted to the disk image, yielding a position angle of $356^{\circ} \pm 5^{\circ}$ and a ratio of the minor to major axes of $0.63 \pm 0.04$. If, as is likely for dust orbiting 


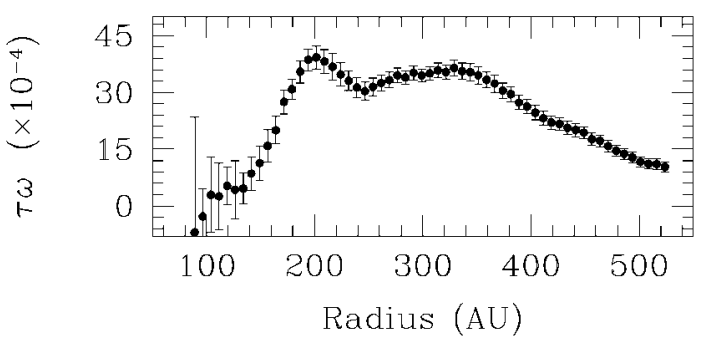

FIG. 3.-The surface density of scatterers plotted as a function of radius computed by multiplying the surface brightness by $4 \pi r^{2}$ and normalizing to the total stellar flux density.

a star, the disk is intrinsically circular, the axial ratio implies an inclination of $51^{\circ} \pm 3^{\circ}$ from face-on. This geometry was used to "deproject" the disk to a face-on view (see Fig. 1, right).

The azimuthally averaged disk surface brightness, shown in Figure 2 as computed from the deprojected image, peaks at 0.3 $\mathrm{mJy} \operatorname{arcsec}^{-2}$ at a radius of $185 \mathrm{AU}$ and falls off to larger and smaller radii. Fitting the slope of the surface brightness with a power law gives $r^{-3.2}$ for $190<r<250 \mathrm{AU}$. At $250 \mathrm{AU}$, an annulus of lower surface brightness, or a "gap" in the disk, can be seen, which is apparent in Figure 3 where the surface brightness has been multiplied by $4 \pi r^{2}$ to determine the surface density of scatterers (see $\$ 5.2$ ). On either side of this gap, the surface density of scatterers does not vary appreciably. At $r \gtrsim 340 \mathrm{AU}$, the surface brightness of the disk falls off more steeply, as $r^{-5.9}$.

The disk flux density, in a rectangle of area $63 \mathrm{arcsec}^{2}$ outside of 0.6 from the star and extending to 5".3 from the star along the major axis of the disk, is $7.2 \pm 1.8 \mathrm{mJy}$. This integration excluded pixels in the diffraction spikes; we make a purely geometric correction by finding the average flux density at every radius and multiplying it by the number of excluded pixels. The corrected total flux density is $8 \pm 2 \mathrm{mJy}$. The F110W magnitudes of 49 Cet and HD 141569 were extrapolated from their $H$-band magnitudes, and the uncertainty in their flux ratio completely dominates the uncertainty in the photometry of the disk. A change of $2 \%$ in the subtracted PSF flux makes an $\sim 20 \%$ difference in the integrated disk flux density.

The eastern side of the disk is $1.5 \pm 0.2$ times brighter than the western side. This difference is not an artifact of the PSF subtraction and can be explained by dust particles that preferentially scatter in the forward direction. Such phase functions apply even when the particles are a few times larger than the wavelength of the scattered light (van de Hulst 1957), and they are produced in recent models of fluffy grains (see, e.g., Wolff, Clayton, \& Gibson 1998). Small particles probably do exist in the HD 141569 disk (see § 5.2). The brighter side, therefore, is inclined toward us, and the ratio of forward to backward scattering implies an asymmetry parameter $g$ of 0.11 in the analytic phase function of Henyey \& Greenstein (1941). This is consistent with the results of Augereau et al. (1999b) on HR 4796A in which the upper limit for $g$ was 0.15 .

\section{DISCUSSION}

\subsection{Disk Morphology}

The surface brightness of the disk has a complicated radial morphology that cannot be represented by a single power law. The density of scatterers, assuming the disk is everywhere

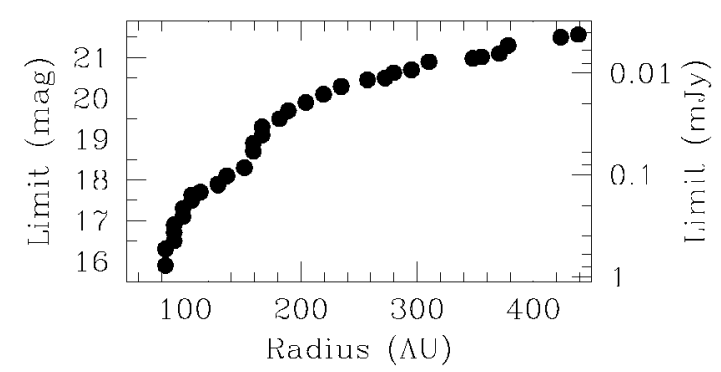

FIG. 4.-Apparent F110W limiting magnitude of an unseen companion as a function of distance from the star. The uncertainty in the detection limit is the size of the points, or $\sim 10 \%$.

optically thin, is as high at radii up to about $360 \mathrm{AU}$ from the star as it is in the region $200 \mathrm{AU}$ from the star.

Dynamical processes could play a large part in shaping the disk. HD 141569 may be the brightest member of a multiple star system, since two bright stars (hereafter B and C) with $H=8.6$ and $9.4 \mathrm{mag}$ lie 7.57 ( $2750 \mathrm{AU})$ and $8.93(\gtrsim 900$ AU) away at respective position angles of $311^{\circ} .5$ and $310^{\circ} .0$. The high probability that these stars are physical companions and assessments of their ages will be discussed in a separate paper (Weinberger et al. 1999). If the three stars are physically associated, the two companions would likely have a significant dynamical effect on the disk. If the companion stars are in the plane of the disk, the physical separation of A and B is $990 \mathrm{AU}$ and that of B and C is $190 \mathrm{AU}$. Then the ratio of the semimajor axes of the wider to the closer pair would only be $\sim 5.2$. This is not expected to be a stable triple system (Eggleton $\&$ Kiseleva 1995), although such young stars may not yet have had time to become unbound.

If the companions are out of the disk plane, they could be much farther from the primary, and their dynamical effect could be to excite significant velocities in the disk dust perpendicular to the plane. This would puff up the disk and possibly flare it. Increased collision velocities could promote the breakup of larger particles into smaller ones and could help account for the large number of scatterers far out in the disk.

The dip in surface brightness at $r \approx 250 \mathrm{AU}$, which is most easily seen along the major axis (Fig. 1), is a striking feature of the disk. The full width at half-minimum of this gap is 60 AU, to which the intrinsic size of the NICMOS PSF contributes $\sim 5 \%$. The depth of this dip in terms of the density of scatterers ( $\tau \omega$; Fig. 3 ) is $\sim 14 \%$ of the average value between 185 and 340 AU.

No point source is seen within the disk, and to constrain the luminosities of possible companion bodies, we planted PSF "stars," generated with Tiny Tim (Krist \& Hook 1997), at random locations in an image created by substracting the coronagraphic images taken at the two telescope roll angles. The limiting magnitude $(3 \sigma)$ of an object that could be detected at each radius is plotted in Figure 4. This limit does not apply to the region obscured by the diffraction spikes that, at the radius of the gap, hide $\sim 17 \%$ of the disk.

At the radius of the gap, the limiting magnitude of 20.3 and an assumed age $\leq 10 \mathrm{Myr}$ correspond to a planet with a mass $\leq 0.003 M_{\odot}\left(3 M_{\mathrm{J}}\right)$ according to the cooling curves by Burrows et al. (1997). At this age, the brown dwarf-planet transition occurs at $\log L / L_{\odot} \approx-3$, which corresponds to an F110W magnitude of $\sim 16$. Figure 4 shows that either a star or a brown dwarf would be clearly detectable in our image at any radii greater than $\sim 80 \mathrm{AU}$. 
If the gap is cleared only by the gravity of a co-orbital planet, the planet mass is $M / M_{\odot}=C(\Delta a / a)^{3}$, where $\Delta a$ and $a$ are the width and radius of the gap, respectively, and the constant $C \approx 0.1$ (Lissauer 1993). This corresponds to a planetary mass of $\sim 1.3$ jupiters, which, given the detection limits discussed in $\S 5.1$, could not have been detected. The orbital period for material $250 \mathrm{AU}$ from the $2.3 M_{\odot}$ star is $2600 \mathrm{yr}$. If we assume that it takes $\gtrsim 300$ orbital periods to clear material in a gap, based on models of disks by Bryden et al. (1999), then a gap can be opened in $\gtrsim 8 \times 10^{5}$ yr. That the gap is not completely clear may mean that processes are moving particles through the disk. The Poynting-Robertson timescale for particles $\geq 1 \mu \mathrm{m}$ is $\sim 4 \times 10^{6} \mathrm{yr}$, similar to the age of the star. Radiation pressure will preferentially clear small grains on a timescale of $\sim 10^{4} \mathrm{yr}$ and could fill in a gap if such grains are present, or being created, in the disk.

\subsection{Constituent Grains}

We can combine the information we infer from the NICMOS images with what is known about the disk from mid- to farinfrared measurements. For reflection from optically thin dust, $\tau \omega=4 \pi \phi^{2}(S / F)$, where $\phi$ is the angular distance of the scatterers from the illuminating star, $S$ is surface brightness, $F$ is the received flux from the star, $\tau$ is the optical depth of the scatterers, and $\omega$ is the albedo (Fig. 3). At the peak surface brightness, $\phi=1^{\prime \prime} .9, S=0.3 \mathrm{mJy} \operatorname{arcsec}^{-2}$, and $\tau \omega=4 \times$ $10^{-3}$. The optical depth to visual absorption implied by the far infrared excess is $8.4 \times 10^{-3}$; if this is also the absorption optical depth at $1.1 \mu \mathrm{m}$, then the albedo of the scatterers is $\sim 0.3$. However, about one-third of the luminosity emitted from 3.5 to $25 \mu \mathrm{m}$ arises from a region within $150 \mathrm{AU}$ of the star (Silverstone et al. 1999). So the total infrared $\tau$ overestimates the optical depth in the exterior part of the disk seen in reflected light and results in an underestimate of the albedo. In the region at $\sim 1 " .9$ (190 AU), therefore, the albedo is greater than 0.3 and probably more like 0.4 . This is a substantially higher albedo than the zodiacal dust in our own solar system (0.1; Dumont \& Levasseur-Regourd 1988) but is similar to the albedos of mineral mixtures and ices found in Infrared Space Observatory spectra of Herbig Ae/Be disks (Malfait et al. 1998).

At $r<150 \mathrm{AU}$, the dust temperature is $\sim 150 \mathrm{~K}$ as estimated from mid-infrared colors and luminosity (Silverstone et al. 1999; Fisher et al. 1999). If the grains are icy, the decline in the $1.1 \mu \mathrm{m}$ surface brightness inside $185 \mathrm{AU}$ may be due to the sublimation of the ice at temperatures of $\sim 100 \mathrm{~K}$. In this case, the grains emitting in the mid- to far-infrared are the iceless counterparts of the grains at higher radius.

Gaseous CO was detected by Zuckerman et al. (1995) with a velocity width of $7.6 \mathrm{~km} \mathrm{~s}^{-1}$, corresponding to a radius of 86 AU given a disk inclination of $51^{\circ}$ and a stellar mass of $2.3 M_{\odot}$. The gas therefore is well inside the brightest part of the reflected light disk and the gap. In the spectrum of Sylvester et al. (1996), a large rise in the continuum shortward of $8 \mu \mathrm{m}$ and a possible peak at $11.3 \mu \mathrm{m}$ both indicate emission from polycyclic aromatic hydrocarbons. The existence of gas and small grains in the presence of forces that remove them very quickly indicates that they are constantly being resupplied. The disk seems to be in an active planetesimal building phase.

\section{CONCLUSIONS}

NICMOS images reveal a large circumstellar disk around the Herbig Ae/Be star HD 141569. The structure of the disk, including a region of depleted material at $250 \mathrm{AU}$, can most easily be understood as being due to dynamical sculpting by one or more planets orbiting within it. If planets exist in this system, they must have formed in less than $10^{7} \mathrm{yr}$ and far from the central star compared with the planets in our own solar system.

We thank David Koerner, Andrea Ghez, and Mike Jura for helpful conversations. This work is based on observations with the NASA/ESA Hubble Space Telescope, obtained at the Space Telescope Science Institute, which is operated by the Association of Universities for Research in Astronomy, Inc., under NASA contract NAS2-6555 and supported by NASA grant NAG5-3042 to the NICMOS instrument definition team.

\section{REFERENCES}

Augereau, J. C., Lagrange, A. M., Mouillet, D., \& Menard, F. 1999a, A\&A, in press

Augereau, J. C., Lagrange, A. M., Mouillet, D., Papaloizou, J. C. B., \& Grorod, P. A. 1999 b, A\&A, 348, 557

Bryden, G., Chen, X., Lin, D. N. C., Nelson, R. P., \& Papaloizou, J. C. B. 1999, ApJ, 514, 344

Burrows, A., Marley, M., Hubbard, W. B., Lunine, J. I., Guillot, T., Saumon, D., Freedman, R., \& Sharp, C. 1997, ApJ, 491, 856

Dumont, R., \& Levasseur-Regourd, A. C. 1988, A\&A, 191, 154

Eggleton, P., \& Kiseleva, L. 1995, ApJ, 455, 640

Fisher, R. S., Telesco, C. M., Piña, R. K., Knacke, R. F., \& Wyatt, M. C. 1999, ApJ, submitted

Henyey, L. G., \& Greenstein, J. L. 1941, ApJ, 93, 70

Jaschek, C., \& Jaschek, M. 1992, A\&AS, 95, 535

Jaschek, M., Jaschek, C., \& Egret, D. 1986, A\&A, 158, 325

Jura, M., Malkan, M., White, R., Telesco, C., Piña, R., \& Fisher, R. S. 1998, ApJ, 505, 897

Krist, J. E., \& Hook, R. N. 1997, in The 1997 HST Calibration Workshop, with a New Generation of Instruments, ed. S. Casertano (Baltimore: STScI), 192

Kulkarni, V. P., Hill, J. M., Schneider, G., Weymann, R. J., Storrie-Lombardi, L. J., Rieke, M. J., Thompson, R. I., \& Januzzi, B. 1999, ApJ, submitted
Lissauer, J. J. 1993, ARA\&A, 31, 129

McLeod, B. A. 1997, in The 1997 HST Calibration Workshop, with a New Generation of Instruments, ed. S. Casertano (Baltimore: STScI), 281

Malfait, K., Waelkens, C., Waters, L. B. F. M., Vandenbussche, B., Huygen, E., \& de Graauw, M. S. 1998, A\&A, 332, L25

Sahu, M. S., Blades, J. C., He, L., Hartmann, D., Barlow, M. J., \& Crawford, I. A. 1998, ApJ, 504, 522

Schneider, G. 1998, in NICMOS and the VLT, ed. W. Freudling \& R. N. Hook (Garching: ESO), 88

Silverstone, M. D., Marsh, K. A., Becklin, E. E., Zuckerman, B., Koerner, D. W., Werner, M. W., \& Ressler, M. E. 1999, in preparation

Sylvester, R. J., Skinner, C. J., Barlow, M. J., \& Mannings, V. 1996, MNRAS, 279,915

van de Hulst, H. C. 1957, Light Scattering by Small Particles (New York: Wiley)

van den Ancker, M. E., de Winter, D., \& Tjin A Djie, H. R. E. 1998, A\&A, 330,145

Weinberger, A. J., et al. 1999, in preparation

Wolff, M. J., Clayton, G. C., \& Gibson, S. J. 1998, ApJ, 503, 815

Zuckerman, B., Forveille, T., \& Kastner, J. H. 1995, Nature, 373, 494 